\title{
Association between shunt-responsive idiopathic normal pressure hydrocephalus and alcohol
}

\author{
${ }^{*}$ Thu-Trang Hickman, MPH, ${ }^{1}$ Matthew E. Shuman, BA,, Tatyana A. Johnson, ${ }^{1}$ Felix Yang, BS, ${ }^{1}$ \\ Rebecca R. Rice, ${ }^{1}$ Isaac M. Rice, ${ }^{1}$ Esther H. Chung, BA, ${ }^{1}$ Robert Wiemann, BA, ${ }^{1}$ Megan Tinl, DPT, ${ }^{1,2}$ \\ Christine Iracheta, PT, ${ }^{1,2}$ Grace Chen, DPT, 1,2 Patricia Flynn, PT, ${ }^{1,2}$ Mary Beth Mondello, NP, MSN, ${ }^{1}$ \\ Jillian Thompson, ${ }^{1}$ Mary-Ellen Meadows, PhD, ${ }^{1,3}$ Rona S. Carroll, PhD, ${ }^{1}$ \\ Hong Wei Yang, MD, PhD, ${ }^{1}$ Hongyan Xing, PhD, ${ }^{1}$ David Pilgrim, MD, ${ }^{1,3}$ E. Antonio Chiocca, MD, PhD, ${ }^{1}$ \\ Ian F. Dunn, MD, ${ }^{1}$ Alexandra J. Golby, MD, ${ }^{1}$ and Mark D. Johnson, MD, PhD ${ }^{1}$
}

\begin{abstract}
${ }^{1}$ Adult Hydrocephalus Program, Department of Neurosurgery, Brigham and Women's Hospital and Harvard Medical School; 'Department of Rehabilitation Services, Brigham and Women's Hospital; and 'Department of Neurology, Brigham and Women's Hospital and Harvard Medical School, Boston, Massachusetts
\end{abstract}

\begin{abstract}
OBJECTIVE Idiopathic normal pressure hydrocephalus (iNPH) is characterized by ventriculomegaly, gait difficulty, incontinence, and dementia. The symptoms can be ameliorated by CSF drainage. The object of this study was to identify factors associated with shunt-responsive iNPH.

METHODS The authors reviewed the medical records of 529 patients who underwent shunt placement for iNPH at their institution between July 2001 and March 2015. Variables associated with shunt-responsive iNPH were identified using bivariate and multivariate analyses. Detailed alcohol consumption information was obtained for 328 patients and was used to examine the relationship between alcohol and shunt-responsive iNPH. A computerized patient registry from 2 academic medical centers was queried to determine the prevalence of alcohol abuse among $1665 \mathrm{iNPH}$ patients.

RESULTS Bivariate analysis identified associations between shunt-responsive iNPH and gait difficulty (OR 4.59, 95\% $\mathrm{Cl} 2.32-9.09 ; p<0.0001$ ), dementia (OR 1.79, 95\% Cl 1.14-2.80; $p=0.01$ ), incontinence (OR 1.77, 95\% Cl 1.13-2.76; $p$ $=0.01)$, and alcohol use (OR 1.98, 95\% Cl 1.23-3.16; $p=0.03)$. Borderline significance was observed for hyperlipidemia (OR 1.56, 95\% Cl 0.99-2.45; $p=0.054$ ), a family history of hyperlipidemia (OR $3.09,95 \% \mathrm{Cl} 0.93-10.26, p=0.054$ ), and diabetes (OR $1.83,95 \% \mathrm{Cl} 0.96-3.51 ; \mathrm{p}=0.064)$. Multivariate analysis identified associations with gait difficulty (OR $3.98,95 \% \mathrm{Cl} 1.81-8.77 ; \mathrm{p}=0.0006)$ and alcohol $(\mathrm{OR} 1.94,95 \% \mathrm{Cl} 1.10-3.39 ; \mathrm{p}=0.04)$. Increased alcohol intake correlated with greater improvement after CSF drainage. Alcohol abuse was 2.5 times more prevalent among iNPH patients than matched controls.
\end{abstract}

CONCLUSIONS Alcohol consumption is associated with the development of shunt-responsive iNPH.

https://thejns.org/doi/abs/10.3171/2016.6.JNS16496

KEY WORDS normal pressure hydrocephalus; gait disorders; incontinence; cognitive impairment; aging; alcohol

$\mathrm{I}$ DIOPATHIC normal pressure hydrocephalus (iNPH) is a neurological disorder that is characterized by gait instability, dementia, and urinary incontinence. ${ }^{1,15} \mathrm{Im}$ aging findings include enlarged cerebral ventricles, deep white matter or periventricular white matter abnormalities, and alterations in the size of the subarachnoid spaces ${ }^{40}$ It has been estimated that about $1.4 \%$ of patients older than 65 years and up to $14 \%$ of patients in nursing homes have iNPH, most of whom are undiagnosed. $6,20,27,36$ Because placement of a shunt to drain the CSF can improve the symptoms,${ }^{14,22,37}$ iNPH has been classified as one of the reversible dementias.
The etiology of shunt-responsive iNPH is unknown. However, clinical studies of iNPH have led to multiple hypotheses regarding its origins. Abnormalities of the cerebral vasculature and defects in CSF circulation are among the leading candidates. In support of the vascular hypothesis, several studies have reported an increased association of vascular risk factors with shunt-responsive iNPH., $, 9,11,16$, 18,21,23,24,33,34 Hypertension, diabetes, and cardiac disease have all been associated with shunt-responsive iNPH. ${ }^{24}$ Periventricular and deep white matter lesions are often seen on MRI in patients with shunt-responsive iNPH and are thought by many to represent cerebral small vessel dis-

ABBREVIATIONS $\mathrm{iNPH}=$ idiopathic normal pressure hydrocephalus; TUG = Timed Up and Go. SUBMITTED February 26, 2016. ACCEPTED June 8, 2016.

INCLUDE WHEN CITING Published online September 30, 2016; DOI: 10.3171/2016.6.JNS16496.

* Mrs. Hickman and Mr. Shuman contributed equally to this work. 
ease. ${ }^{40}$ In fact, patients with Binswanger's cerebrovascular encephalopathy can improve after CSF drainage. ${ }^{33,37}$

Disorders of CSF absorption have also been postulated as causes of shunt-responsive iNPH. Perhaps the strongest evidence in favor of this hypothesis is that ventricular enlargement is a hallmark of shunt-responsive iNPH, and CSF drainage improves the symptoms. . $14,15,22,37,40$ In addition, studies have suggested the presence of impaired CSF circulation and abnormal subarachnoid spaces in shuntresponsive iNPH patients, providing additional support for the CSF hypothesis. ${ }^{3}$

It is unclear whether behavioral or environmental factors contribute to the development of shunt-responsive iNPH. Here, we report a correlation between the development of shunt-responsive iNPH and increased alcohol consumption.

\section{Methods \\ Study Design and Data Collection}

The study was conducted under the auspices of a human subjects research protocol approved by the Partners Institutional Review Board Committee. We retrospectively reviewed the medical records of patients diagnosed with disorders of CSF flow at Brigham and Women's Hospital between July 2001 and May 2015 to identify patients who had undergone evaluation for iNPH. Patients were selected for evaluation of possible iNPH if they were found to have communicating hydrocephalus on CT or MR images, gait disturbance, urinary urgency/incontinence, cognitive impairment, and no clearly identifiable cause of their symptoms. At the time of the first clinic visit, clinical and demographic data were collected using a patient questionnaire. We combined these prospectively collected data with additional information retrieved from an electronic medical record.

We also identified patients for whom detailed information on the amount of alcohol consumed was available. Using dietary recommendations published by the US Department of Health and Human Services and the US Department of Agriculture as a guide, ${ }^{39}$ we divided alcohol consumption into 4 categories: never (abstinence), rare/ occasional ( 3 alcoholic beverages or fewer weekly), moderate (4-14 alcoholic beverages weekly) and heavy (more than 14 alcoholic beverages weekly). A guideline that takes into account the lower threshold for heavy drinking among women of more than 1 drink/day was also used..$^{39}$

To determine the prevalence of alcohol abuse among a larger iNPH cohort, we queried a computerized, diagnostic code-based patient registry (Partners Healthcare System Research Patient Data Registry). The query, which was performed in February 2015, searched the medical records of 3,853,907 patients from 2 large academic medical centers (Brigham and Women's Hospital and Massachusetts General Hospital) using the diagnostic codes for iNPH and alcohol abuse not otherwise specified. A control group matched for age, sex, and race was assembled for comparison.

\section{Outcome Assessment}

Patients were evaluated by a group of board-certified neurosurgeons and/or neurologists before and after CSF drainage trials and shunt placement. Quantitative and qualitative assessments of Timed Up and Go (TUG) tests, Tinetti balance tests, and other evaluations of gait and balance were performed by physical therapists during a 3-day CSF drainage trial. Additional information regarding changes in gait, cognition, and urinary or fecal continence was obtained from patient and caregiver reports.

Patients who showed signs of improvement in gait, incontinence, or cognition after a trial of CSF drainage were offered ventriculoperitoneal shunt placement. Patients were evaluated postoperatively at 2 weeks and at 6 weeks, and were then followed with periodic monthly or annual clinic visits as needed for a period of up to 11 years. Changes in gait were based on assessments of TUG time, gait speed, step height, cadence, balance, the need for gait assistive devices, and the number of falls. Changes in urinary/fecal incontinence or urgency were assessed in collaboration with nursing staff, caregivers, and patients. Urinary and fecal incontinence were assessed via written questionnaire and via daily inquiries of nursing staff, patients, and caregivers during the extended trial of lumbar CSF drainage and during preoperative and postoperative clinic visits. Changes in cognitive function were assessed using neurological examinations, formal neurocognitive evaluations (including the Montreal Cognitive Assessment, Mini-Mental State Examination, and evaluations by a licensed cognitive psychologist), statements by caregivers, and patient self-reports. These criteria were collectively used to categorize the degree of improvement within 1 year of shunt placement as markedly improved, mildly improved, or not improved. The combined criteria for not improved were 1) no improvement in ambulation; 2) no improvement in gait speed or TUG time; 3) no decrease in the number of falls; 4) no improvement in incontinence or urgency; 5) no improvement in the need for assistance with activities of daily living; 6) no improvement in reasoning, cognitive, or conversational abilities; and 7) no improvement in memory. The combined criteria for mild improvement were 1) progression from the use of one gait assistive device to a lesser device; 2) less than a $10 \%$ improvement in gait speed or TUG time; 3) a decrease in the number of falls; 4) less than a $50 \%$ decrease in episodes of incontinence or urgency; 5) decreased need for assistance with activities of daily living, but no change in independence; 6) mild improvement in reasoning, cognitive, or conversational abilities; and 7) mild improvement in memory. The combined criteria for marked improvement were 1) progression to ambulation without assistance; 2) greater than a $10 \%$ improvement in gait speed or TUG time; 3) complete cessation of falls; 4) greater than a $50 \%$ decrease in episodes of incontinence or urgency; 5) progression from dependent to assisted living, or from assisted to independent living; 6) marked improvement in reasoning, cognitive, or conversational abilities; and 7) marked improvement in memory.

\section{Ventriculoperitoneal Shunt Surgery}

Patients for whom a trial of CSF drainage supported a diagnosis of probable iNPH underwent surgical implantation of a ventriculoperitoneal shunt as treatment for their 


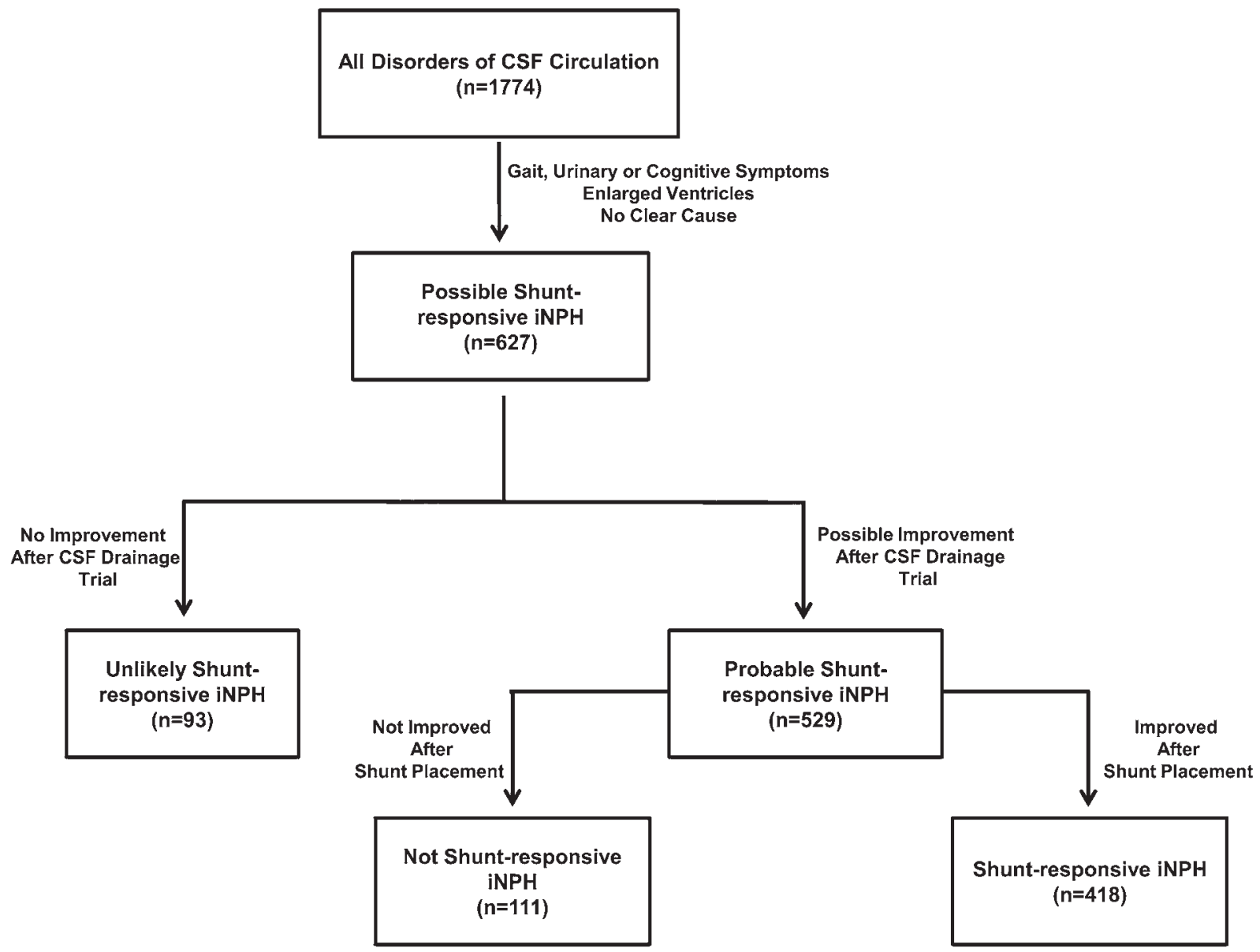

FIG. 1. Study design. Flow diagram depicting the design for the iNPH study.

symptoms. The shunts generally contained programmable valves (Codman, Inc.) programmed initially to a setting of $120 \mathrm{~mm} \mathrm{H}_{2} \mathrm{O}$. Valve settings were adjusted postoperatively as needed to maximize symptom improvement and minimize signs or symptoms of overdrainage, such as positional headaches or subdural hematomas.

\section{Statistical Analysis}

For statistical analysis, we used the SAS statistical software program (version 9.3, SAS Institute). Bivariate analysis was used to determine whether there were significant differences in presenting symptoms or the presence of clinical or demographic factors between patients who improved after shunt placement and those who did not improve. For medical history or family history variables, the presence or absence of disease documentation in the medical record was used to categorize patients as affected or unaffected, respectively. Consistent with clinical practice, medical history or family history variables were treated as binary and were marked as positive only when they were found in the patients' medical records. For analysis of tobacco or alcohol use, patients were divided into users, nonusers, and those for whom no information was available. The chi-square test of homogeneity was used to determine statistical significance, and $\mathrm{p} \leq 0.05$ was set as the threshold. A multivariate logistic regression analysis was used to investigate the association between the variables considered and improvement after shunt placement. The independent predictor variables included presenting clinical symptoms, medical history, family history, tobacco and alcohol use, age, and sex. Statistical significance was set at the $\mathrm{p} \leq 0.05$ level.

For the analysis of data regarding the prevalence of alcohol use among patients with iNPH, statistical significance was determined using the proportion test. A z-score was calculated based on a 2-tailed hypothesis, and significance was set at $\mathrm{p} \leq 0.05$. All statistical analyses were performed by 2 authors (T.T.H. and M.J).

\section{Results}

\section{Patient Population}

We identified 622 patients who presented with clinical symptoms and imaging findings consistent with iNPH. After an evaluation that included history taking, neurological examination, cranial imaging, and a trial of CSF drainage, 529 patients (267 men and 262 women) were suspected to have iNPH and underwent shunt placement (Fig. 1). The median and mean duration of symptoms prior to evaluation for iNPH were 24 months and $29.6 \pm 20.9$ months (mean $\pm \mathrm{SD}$ ), respectively. More than $95 \%$ of the patients who underwent shunt placement presented with 
gait difficulty as one of their primary symptoms. About $63 \%$ of patients had the classic iNPH symptom triad of gait difficulty, urinary incontinence/urgency, and cognitive impairment.

The mean follow-up duration after shunt placement was $32.3 \pm 30.8$ months, with a range of 1-132 months (median 22 months). Of the 529 patients who underwent shunt placement, 418 (79\%) improved (shunt-responsive iNPH group) and 111 (21\%) did not improve (not shunt-responsive iNPH group). Approximately $78 \%$ of patients who presented with the iNPH symptom triad improved after shunt placement. Patients who presented with gait difficulty alone $(8 \%)$ or in combination with either incontinence $(11 \%)$ or cognitive deficits $(17 \%)$ improved about $60 \%$ of the time after CSF drainage. Demographic and clinical characteristics of the patients who improved after shunt placement and those who did not are shown in Table 1.

\section{Factors Associated With Shunt-Responsive iNPH}

Bivariate analysis identified several factors that are associated with the presence of shunt-responsive iNPH (Fig. 2). Presenting symptoms associated with shunt-responsive iNPH included gait difficulty (OR 4.59, CI 2.32-9.09; p $<0.0001$ ), cognitive dysfunction (OR 1.79, CI 1.14-2.80; $\mathrm{p}=0.01)$ and urinary urgency/incontinence (OR 1.77, CI 1.13-2.76; $\mathrm{p}=0.01$ ). Among comorbid conditions, positive trends toward significance were observed for hyperlipidemia (OR 1.56, CI 0.99-2.45; $\mathrm{p}=0.054)$ and diabetes (OR 1.83 , CI $0.96-3.51 ; p=0.064)$. In addition, a positive trend toward significance was seen for a family history of hyperlipidemia (OR 3.09, CI 0.93-10.26; $\mathrm{p}=0.054$ ). A positive association between alcohol use and the presence of shuntresponsive iNPH was also observed (OR 1.97, CI 1.233.16; $\mathrm{p}<0.03$ ). We did not observe significant associations between iNPH and hypertension (OR 1.23, CI 0.79-1.90; p $=0.36)$ or smoking $(\mathrm{OR} 0.99$, CI 0.62-1.56; $\mathrm{p}=0.95)$.

For multivariate analysis, we used a more parsimonious model containing 15 variables (Fig. 3). This analysis identified positive associations between the presence of shunt-responsive iNPH and gait difficulty (OR 3.98, CI 1.81-8.77; $\mathrm{p}<0.0006)$ or alcohol consumption (OR 1.94, 95\% CI 1.10-3.39; $\mathrm{p}=0.04)$.

\section{Alcohol and Shunt-Responsive iNPH}

We performed further analysis of 328 patients (162 men and 166 women) for whom detailed information regarding the amount of alcohol consumed was available. Overall, $47 \%$ of these patients consumed alcohol to some degree. This prevalence was comparable to the prevalence of alcohol use of $46 \%$ observed among a group of 152 brain tumor patients 60 years and older identified at our institution. However, 54\% of patients who improved after CSF drainage consumed alcohol, while only $26 \%$ of those who did not improve consumed alcohol (z-score $=-3.94$, $\mathrm{p}<0.0001$ ).

A positive relationship between increased alcohol consumption and the probability of having shunt-responsive iNPH was observed. After CSF drainage, approximately $63 \%$ of nondrinkers improved, $82 \%$ of rare/occasional drinkers improved, $87 \%$ of moderate drinkers improved,
TABLE 1. Characteristics of groups stratified by outcome after shunt placement for iNPH

\begin{tabular}{|c|c|c|}
\hline Characteristic & Improved & Not Improved \\
\hline Mean age, yrs \pm SD & $74.7 \pm 7.3$ & $74.6 \pm 7.4$ \\
\hline Male sex & $212(50.7 \%)$ & $55(49.6 \%)$ \\
\hline Gait instability & $400(95.7 \%)$ & $92(82.9 \%)$ \\
\hline Cognitive difficulty & $318(76.1 \%)$ & $71(64.0 \%)$ \\
\hline Urinary urgency/incontinence & $314(75.1 \%)$ & $70(63.1 \%)$ \\
\hline Headache & $15(3.6 \%)$ & $3(2.7 \%)$ \\
\hline Hypertension & $283(67.7 \%)$ & $70(63.1 \%)$ \\
\hline Cardiac disease & $69(16.5 \%)$ & $20(18.0 \%)$ \\
\hline Arthritis & $56(13.4 \%)$ & $12(10.8 \%)$ \\
\hline Inflammatory disease & $81(19.4 \%)$ & $20(18.0 \%)$ \\
\hline Renal disease & $26(6.2 \%)$ & $5(4.5 \%)$ \\
\hline Asthma & $27(6.5 \%)$ & $4(3.6 \%)$ \\
\hline Diabetes & $76(18.2 \%)$ & $12(10.8 \%)$ \\
\hline Hypothyroidism & $45(10.8 \%)$ & $10(9.0 \%)$ \\
\hline Hyperthyroidism & $8(1.9 \%)$ & $1(0.9 \%)$ \\
\hline All thyroid disease & $66(15.8 \%)$ & $13(11.7 \%)$ \\
\hline Trauma & $3(0.7 \%)$ & $1(0.9 \%)$ \\
\hline Cancer & $92(22.0 \%)$ & $21(18.9 \%)$ \\
\hline Fracture & $10(2.4 \%)$ & $1(0.9 \%)$ \\
\hline Hyperlipidemia & $166(39.7 \%)$ & $33(29.7 \%)$ \\
\hline Family $\mathrm{Hx}$ of cancer & $77(18.4 \%)$ & $18(16.2 \%)$ \\
\hline Family Hx of Alzheimer's disease & $11(2.6 \%)$ & $3(2.7 \%)$ \\
\hline Family Hx of Parkinson's disease & $11(2.6 \%)$ & $3(2.7 \%)$ \\
\hline Family Hx of hypertension & $58(13.9 \%)$ & $10(9.0 \%)$ \\
\hline Family Hx of stroke & $16(3.8 \%)$ & $3(2.7 \%)$ \\
\hline Family Hx of hyperlipidemia & $33(7.9 \%)$ & $3(2.7 \%)$ \\
\hline Family $\mathrm{Hx}$ of cardiac disease & $73(17.5 \%)$ & $20(18.0 \%)$ \\
\hline Family $\mathrm{Hx}$ of renal disease & $1(0.2 \%)$ & $1(0.9 \%)$ \\
\hline Family $\mathrm{Hx}$ of asthma & $3(0.7 \%)$ & $0(0.0 \%)$ \\
\hline Family $\mathrm{Hx}$ of dementia & $16(3.8 \%)$ & $6(5.4 \%)$ \\
\hline Family $\mathrm{Hx}$ of gait difficulty & $1(0.2 \%)$ & $1(0.9 \%)$ \\
\hline Smoking & $123(29.4 \%)$ & $33(29.7 \%)$ \\
\hline Alcohol use & $166(39.7 \%)$ & $30(27.0 \%)$ \\
\hline Recreational drug use & $3(0.7 \%)$ & $1(0.9 \%)$ \\
\hline
\end{tabular}

$\mathrm{Hx}=$ history.

and $90 \%$ of heavy drinkers improved (z-score $=-2.57, \mathrm{p}$ $<0.01$; Fig. 4A). The mean age for drinkers $(74.7 \pm 7.3$ years) and nondrinkers ( $73.7 \pm 8.6$ years) was not significantly different. However, the female to male ratio was 1:1.7 for drinkers and 0.83:1 for nondrinkers, consistent with studies demonstrating a higher prevalence of alcohol use among men. ${ }^{19}$ When the data for men and women were analyzed separately using sex-specific guidelines for heavy drinking (i.e., more than 1 drink daily for women and 2 drinks daily for men), a positive relationship between increased alcohol consumption and the probability of having shunt-responsive iNPH was seen for both men and women (Fig. 4B).

At the time of database construction and prior to statistical analysis, patient responses after shunt placement were 


\begin{tabular}{|c|c|c|c|c|c|}
\hline \multirow[b]{2}{*}{ Gender } & 0.1 & \multirow{2}{*}{$\begin{array}{c}\text { Odds Ratio } \\
0.9543\end{array}$} & \multicolumn{2}{|c|}{ 95\% Confidence Interval } & \multirow{2}{*}{$\begin{array}{r}P \text { value } \\
0.8268\end{array}$} \\
\hline & $\mathbf{r} \boldsymbol{H}$ & & 0.6279 & 1.4504 & \\
\hline Gait Difficulty & $\longmapsto-1$ & 4.5894 & 2.3173 & 9.0892 & $<.0001$ \\
\hline Cognitive Difficulty & $1+\bullet-1$ & 1.7915 & 1.1447 & 2.8039 & 0.0101 \\
\hline Urgency/Incontinence & $\mid 1-\bullet$ & 1.7684 & 1.1336 & 2.7587 & 0.0114 \\
\hline Confusion & & 1.0146 & 1.0029 & 1.0264 & 0.2043 \\
\hline Headache & $\vdash$ & 1.34 & 0.381 & 4.7128 & 0.6472 \\
\hline Hypertension & He- & 1.2278 & 0.7935 & 1.9 & 0.3564 \\
\hline Cardiac Disease & & 0.8996 & 0.5197 & 1.557 & 0.7052 \\
\hline Arthritis & $\mapsto$ & 1.2762 & 0.6584 & 2.474 & 0.4693 \\
\hline Inflammatory Disease & $\mapsto-1$ & 1.0769 & 0.6264 & 1.8515 & 0.7886 \\
\hline Renal Disease & $\stackrel{1}{1}-1$ & 1.4061 & 0.5273 & 3.7498 & 0.4939 \\
\hline Asthma & $\stackrel{1}{\longrightarrow} \bullet$ & 1.8472 & 0.6326 & 5.394 & 0.2548 \\
\hline Diabetes & $\mapsto-1$ & 1.8333 & 0.9584 & 3.507 & 0.0638 \\
\hline Hypothyroidism & $\stackrel{-10-1}{ }$ & 1.2185 & 0.5933 & 2.5024 & 0.5899 \\
\hline All thyroid disease & $\stackrel{1}{1} \bullet-1$ & 1.4135 & 0.7487 & 2.6685 & 0.284 \\
\hline Trauma & $\longmapsto$ & 0.7952 & 0.0819 & 7.7193 & 0.843 \\
\hline Cancer & $\operatorname{He-1}$ & 1.2095 & 0.7131 & 2.0514 & 0.48 \\
\hline Hyperlipidemia & -1 & 1.557 & 0.9911 & 2.446 & 0.0536 \\
\hline Family Hx of Cancer & & 1.1667 & 0.6651 & 2.0466 & 0.5906 \\
\hline Family Hx of Alzheimer's & $\longmapsto$ & 0.7922 & 0.2108 & 2.9765 & 0.7296 \\
\hline Family Hx of Parkinson's & $\longmapsto$ & 0.973 & 0.2667 & 3.5492 & 0.9669 \\
\hline Family Hx of Hypertension & $H \bullet-1$ & 1.6272 & 0.8029 & 3.2981 & 0.1733 \\
\hline Family Hx of Stroke & $\longmapsto$ & 1.4328 & 0.41 & 5.0075 & 0.5712 \\
\hline Family Hx of Hyperlipidemia & $\longmapsto$ & 3.0857 & 0.9285 & 10.2554 & 0.0535 \\
\hline Family Hx of Cardiac Disease & $1-$ & 0.9628 & 0.5577 & 1.6619 & 0.8916 \\
\hline Family Hx of Renal Disease & $\longrightarrow-1$ & 0.2638 & 0.0164 & 4.251 & 0.3126 \\
\hline Family Hx of Asthma & b & 1.0072 & 0.9991 & 1.0155 & 0.3707 \\
\hline Family Hx of Dementia & $\longmapsto+1$ & 0.6965 & 0.266 & 1.8237 & 0.4593 \\
\hline Family Hx of Gait Difficulty & $\longrightarrow-1 \quad 1$ & 0.2638 & 0.0164 & 4.251 & 0.3126 \\
\hline Smoking & $1-1$ & 0.9855 & 0.6233 & 1.5583 & 0.9502 \\
\hline Alcohol Use & 1 & 1.9762 & 1.2315 & 3.1586 & 0.0347 \\
\hline
\end{tabular}

FIG. 2. Univariate analysis of variables associated with shunt-responsive iNPH. Data shown are odds ratios and $95 \%$ confidence intervals; $\mathrm{p}$ values were calculated using the chi-square test. $\mathrm{Hx}=$ history. Figure is available in color online only.

categorized as no improvement, mild improvement, or marked improvement using the criteria outlined in Methods. We observed that the prevalence of alcohol use increased as the level of improvement after shunt placement increased (Fig. 4C and D). Importantly, a dose-dependent relationship between the amount of alcohol consumed and the level of improvement observed after shunt placement was seen for both men and women (Fig. 4E).

\section{Hospital-Based Computer Registry Analysis}

To examine the prevalence of hypertension and alcohol use among a larger group of iNPH patients, we queried a hospital-based computer registry of more than 3.8 million patients from our institution and a second large academic medical center, Massachusetts General Hospital. Using the registry, we identified 1665 patients with a diagnosis of iNPH. A control group of 1661 patients matched for age, sex, and race was also assembled. A query using the diagnosis "alcohol abuse NOS" identified 105,188 affected patients. Cross-referencing these groups identified 86
iNPH patients with a diagnosis of alcohol abuse but only 35 control group patients with this diagnosis ( $\mathrm{z}$-score = 4.7096, $\mathrm{p}<0.0001)$.

For comparison, we conducted additional queries to investigate the prevalence among iNPH patients of acute appendicitis and toothache, 2 diagnoses that we hypothesized to be unrelated to iNPH. Approximately 15,387 patients with a diagnosis of acute appendicitis were identified in the registry, and 21,105 patients with a diagnosis of toothache were similarly identified. There was no statistically significant difference in the number of iNPH patients or matched control group patients diagnosed with acute appendicitis (8 patients in each group, $\mathrm{p}<1.0$ ) or toothache $(\mathrm{n}=11$ for $\mathrm{iNPH}, \mathrm{n}=5$ for control group; $\mathrm{z}$-score $=$ $1.5, \mathrm{p}<0.13)$.

\section{Discussion}

\section{Cardiovascular Risk Factors and Shunt-Responsive iNPH}

Previous investigators have reported associations be- 


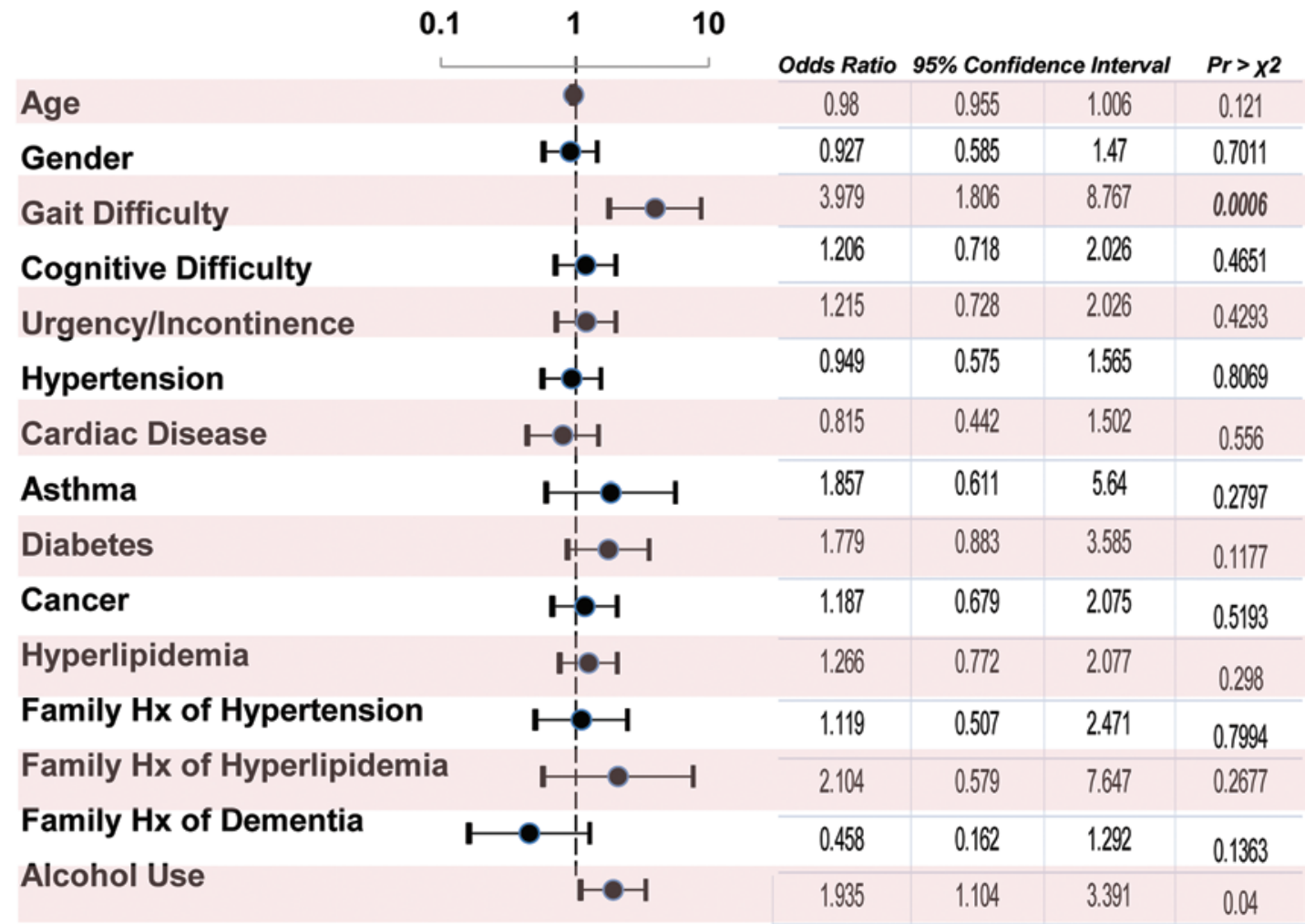

FIG. 3. Multivariate analysis of variables associated with shunt-responsive iNPH. Data shown are odds ratios and $95 \%$ confidence intervals. $\operatorname{Pr}>\chi^{2}$ indicates that chi-square values were obtained using logistic regression analysis. Figure is available in color online only.

tween iNPH and cardiovascular risk factors, such as hypertension, hyperlipidemia, and diabetes. . $^{7,11,16,18,21,23,24,33,34}$ In the current study, bivariate analysis revealed strong positive trends toward significance for hyperlipidemia $(\mathrm{p}$ $=0.054)$, a family history of hyperlipidemia $(\mathrm{p}=0.054)$, and diabetes $(\mathrm{p}=0.064)$. The mechanisms underlying the association between cardiovascular risk factors and shunt-responsive iNPH are not known. One possibility is that hyperlipidemia, diabetes, or other cardiovascular risk factors promote cerebrovascular disease and decreased cerebral blood flow in the deep white matter, and this leads to cognitive and motor dysfunction. Imaging studies have identified microvascular abnormalities in the deep white matter and periventricular areas of the brain in a subpopulation of patients with iNPH. ${ }^{9,18,22,23,25,33,34,40}$ Cerebral blood flow studies have documented decreased cerebral blood flow and decreased cerebrovascular reactivity in iNPH patients, raising the possibility that iNPH is a disease of the cerebral vasculature. Interestingly, some studies have indicated that cerebral blood flow increases and deep white matter lesions decrease in iNPH patients after shunt placement, ${ }^{2,8,29}$ although others have disputed these findings. ${ }^{25}$

Diabetes and hyperlipidemia promote atherosclerosis and have been postulated to play a causal role in mild cognitive impairment. ${ }^{4}$ Arteriosclerotic changes have been identified in postmortem studies of patients with iNPH in about $60 \%$ of cases. ${ }^{26}$ Interestingly, patients with Binswanger's disease (hypertension with subcortical arteriosclerotic disease and dementia) can improve after shunt placement. ${ }^{37}$
The fact that hyperlipidemia and diabetes have been associated with iNPH in multiple studies raises the possibility that abnormal lipid metabolism or cerebrovascular disease may contribute to this disorder.

\section{Alcohol Consumption and Shunt-Responsive iNPH}

Here, we present the first evidence for a robust association between an exposure (alcohol use) and the presence of shunt-responsive iNPH. Even rare alcohol consumption was associated with an increased likelihood of having shunt-responsive iNPH. Moreover, increasing levels of alcohol consumption were associated with increasing degrees of improvement after shunt placement.

It is not possible from this study to infer a causal relationship between alcohol use and the development of shunt-responsive iNPH. In fact, these data are consistent with at least 2 very different hypotheses. One hypothesis is that alcohol use increases the response to CSF drainage in patients who have iNPH. Cerebral blood flow and cerebrovascular reactivity are impaired in iNPH patients, and both are reportedly increased after CSF drainage in patients who improve. ${ }^{2,8,29}$ Alcohol has vasoactive properties, ${ }^{5}$ and numerous studies have shown that alcohol increases cerebral blood flow in a dose-dependent manner. ${ }^{17,35,38}$ Thus, it is tempting to speculate that alcohol enhances the ability of CSF drainage to increase cerebral blood flow, and that this promotes improved outcomes after shunt placement.

An alternative hypothesis, however, is that alcohol promotes the development of shunt-responsive iNPH. In addition to Wernicke-Korsakoff syndrome, heavy alcohol use 


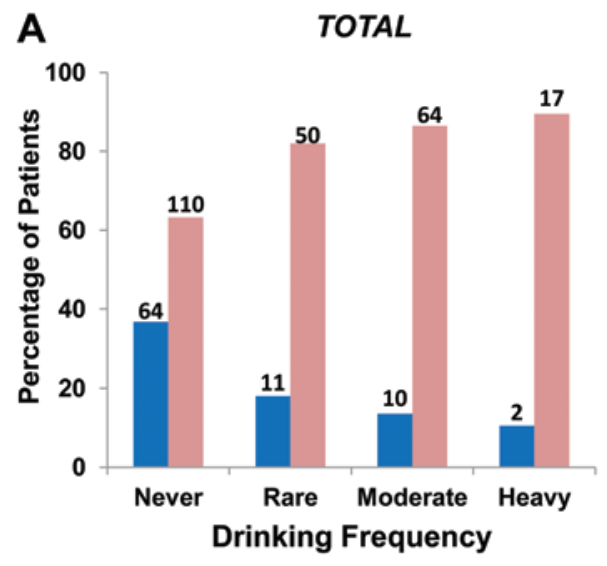

C

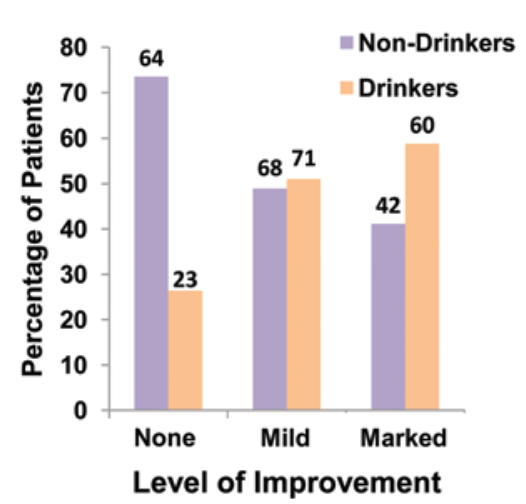

B

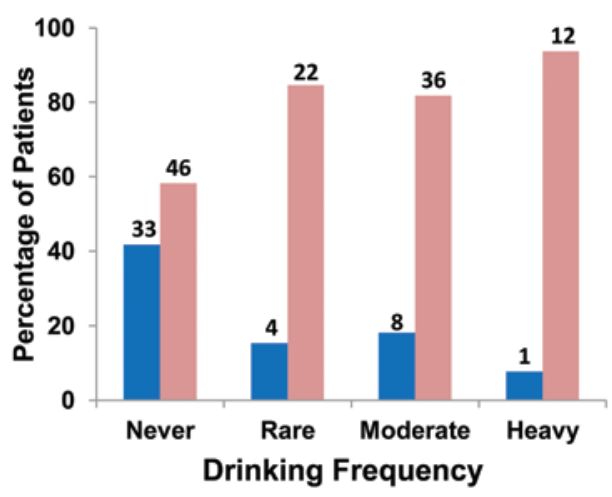

WOMEN

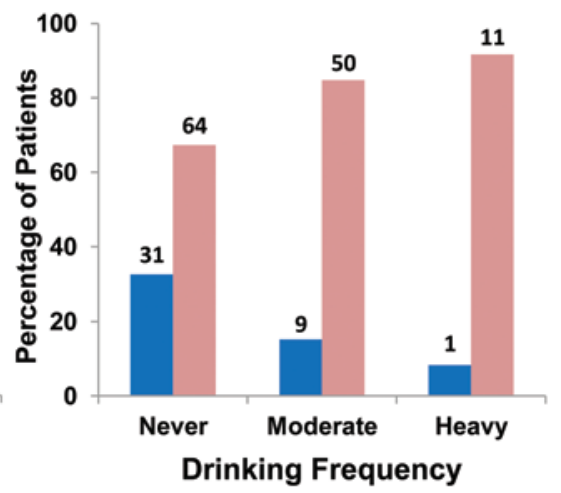

D

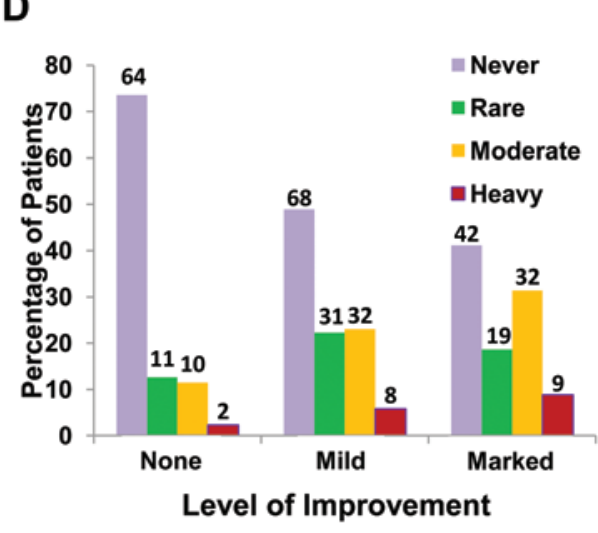

E

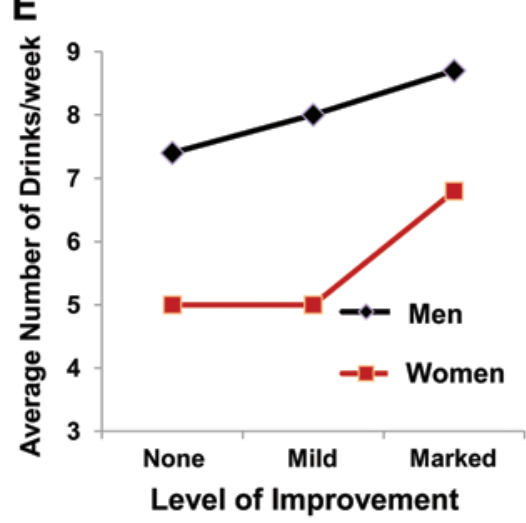

FIG. 4. A: Percentage of patients who improved (red bars) or did not improve (blue bars) after shunt placement for iNPH, grouped according to amount of alcohol consumed. The number of patients involved in each category is shown at the top of each column. Criteria for classifying the frequency of alcohol consumption were as follows: never (abstinence), rare ( 3 alcoholic beverages or less weekly), moderate (4-14 alcoholic beverages weekly), and heavy (more than 14 alcoholic beverages weekly). B: Percentage of men and women who improved (red bars) or did not improve (blue bars) after shunt placement for iNPH, grouped according to frequency of alcohol consumption. Criteria for classifying the frequency of alcohol consumption for men were the same as in panel A. Criteria for classifying the frequency of alcohol consumption for women were never (abstinence), moderate (up to 7 alcoholic beverages weekly), and heavy (more than 7 alcoholic beverages weekly). C: Prevalence of drinkers among patients showing no improvement (none), mild improvement, or marked improvement after CSF drainage for iNPH. The number of patients in each category is shown at the top of each column. D: Graph illustrating relationship between the degree of improvement after CSF drainage for iNPH and the frequency of alcohol consumption. E: Graph showing the relationship between the number of drinks consumed weekly and the degree of improvement for men and women.

can produce alcohol-related dementia. ${ }^{32}$ Alcohol causes injury to cerebral vessels and damage to the deep white matter of the brain, which increases with age. ${ }^{12,13,31}$ Alcohol also disrupts the blood-CSF barrier ${ }^{30}$ and the bloodbrain barrier, ${ }^{10}$ and it decreases cognitive function in animals..$^{10}$ Recent studies in humans have shown that defects in the blood-brain barrier arise during aging and correlate with mild cognitive impairment.$^{28}$ Thus, alcohol-mediated brain injury could act in concert with other aging-related processes to promote the development of shunt-responsive iNPH. Because iNPH is currently identified in part by the positive response to CSF drainage, differentiation between whether alcohol augments the treatment of shunt-responsive iNPH or promotes development of the disease itself will require elucidation of the underlying cause of this disorder.

Selection bias or information bias can adversely affect the interpretation of retrospective studies. In the current study, the selection of patients with iNPH, assessments of the degree of improvement, and the amount of alcohol consumed were all documented prior to initiation of the study. No post hoc diagnoses or assessments were made, and all patients undergoing evaluation for iNPH during the study period were included. This significantly reduces (but does not eliminate) the potential for selection bias.

The retrospective nature of this study prevented us from rigorously controlling outcome assessment or alcohol exposure, and it necessitated that we rely instead on accurate recordkeeping by a number of health care providers during past patient encounters. Even with the large sample size of this study, inaccuracies in recording clinical comorbidities, exposures, or outcomes may have limited our ability to accurately identify associations between iNPH and other variables. In addition, recall bias may derive from inaccuracies in patient reporting of alcohol consumption. Because patients may not have been forthcoming about alcohol use, nonreporting or underreporting of alcohol consumption could minimize the apparent differences in 
iNPH prevalence and outcomes between alcohol users and nonusers in this study.

\section{Conclusions}

The findings reported here reveal an association between alcohol and the development of shunt-responsive iNPH. Previous associations with cardiovascular risk factors, such as diabetes, hyperlipidemia, and hypertension were also identified. The consumption of alcohol is the first exposure associated with shunt-responsive iNPH, and it joins existing associations with cardiovascular risk factors found in this and in previous studies. Accumulating reports indicate that the development of many neurological disorders of aging (e.g., Alzheimer's disease, Parkinson's disease, hearing loss, and mild cognitive impairment) can be influenced by behavior or by environmental exposures. Our study indicates that this may be true for iNPH as well. Prospective studies designed to determine whether alcohol consumption promotes the development of iNPH or, alternatively, whether it increases the response to CSF drainage in patients who already have the disease, should be conducted. The interpretation of such studies would be aided by the discovery of the underlying causes and elucidation of the pathophysiology of iNPH.

\section{Acknowledgments}

This work was supported by a generous gift from Susan and Frederick B. Sontag.

\section{References}

1. Adams RD, Fisher CM, Hakim S, Ojemann RG, Sweet WH: Symptomatic occult hydrocephalus with 'normal' cerebrospinal fluid pressure: a treatable syndrome. N Engl J Med 273:117-126, 1965

2. Akiguchi I, Ishii M, Watanabe Y, Watanabe T, Kawasaki T, Yagi H, et al: Shunt-responsive parkinsonism and reversible white matter lesions in patients with idiopathic NPH. J Neurol 255:1392-1399, 2008

3. Akiguchi I, Shirakashi Y, Budka H, Watanabe Y, Watanabe $\mathrm{T}$, Shiino A, et al: Disproportionate subarachnoid space hydrocephalus-outcome and perivascular space. Ann Clin Transl Neurol 1:562-569, 2014

4. Alagiakrishnan K, Sankaralingam S, Ghosh M, Mereu L, Senior P: Antidiabetic drugs and their potential role in treating mild cognitive impairment and Alzheimer's disease. Discov Med 16:277-286, 2013

5. Altura BM, Altura BT, Gebrewold A: Alcohol-induced spasms of cerebral blood vessels: relation to cerebrovascular accidents and sudden death. Science 220:331-333, 1983

6. Brean A, Eide PK: Prevalence of probable idiopathic normal pressure hydrocephalus in a Norwegian population. Acta Neurol Scand 118:48-53, 2008

7. Casmiro M, D'Alessandro R, Cacciatore FM, Daidone R, Calbucci F, Lugaresi E: Risk factors for the syndrome of ventricular enlargement with gait apraxia (idiopathic normal pressure hydrocephalus): a case-control study. J Neurol Neurosurg Psychiatry 52:847-852, 1989

8. Chang CC, Asada H, Mimura T, Suzuki S: A prospective study of cerebral blood flow and cerebrovascular reactivity to acetazolamide in 162 patients with idiopathic normalpressure hydrocephalus. J Neurosurg 111:610-617, 2009

9. Earnest MP, Fahn S, Karp JH, Rowland LP: Normal pressure hydrocephalus and hypertensive cerebrovascular disease. Arch Neurol 31:262-266, 1974
10. Ehrlich D, Humpel C: Chronic vascular risk factors (cholesterol, homocysteine, ethanol) impair spatial memory, decline cholinergic neurons and induce blood-brain barrier leakage in rats in vivo. J Neurol Sci 322:92-95, 2012

11. Eide PK, Pripp AH: Increased prevalence of cardiovascular disease in idiopathic normal pressure hydrocephalus patients compared to a population-based cohort from the HUNT3 survey. Fluids Barriers CNS 11:19, 2014

12. Fein G, Shimotsu R, Di Sclafani V, Barakos J, Harper C: Increased white matter signal hyperintensities in long-term abstinent alcoholics compared with nonalcoholic controls. Alcohol Clin Exp Res 33:70-78, 2009

13. Fukuda K, Yuzuriha T, Kinukawa N, Murakawa R, Takashima Y, Uchino A, et al: Alcohol intake and quantitative MRI findings among community dwelling Japanese subjects. J Neurol Sci 278:30-34, 2009

14. Gölz L, Ruppert FH, Meier U, Lemcke J: Outcome of modern shunt therapy in patients with idiopathic normal pressure hydrocephalus 6 years postoperatively. J Neurosurg 121:771-775, 2014

15. Graff-Radford NR: Normal pressure hydrocephalus. Neurol Clin 25:809-832,vii-viii, 2007

16. Graff-Radford NR, Godersky JC: Idiopathic normal pressure hydrocephalus and systemic hypertension. Neurology 37:868-871, 1987

17. Gundersen H, van Wageningen H, Grüner R: Alcohol-induced changes in cerebral blood flow and cerebral blood volume in social drinkers. Alcohol Alcohol 48:160-165, 2013

18. Haidri NH, Modi SM: Normal pressure hydrocephalus and hypertensive cerebrovascular disease. Dis Nerv Syst 38:918921, 1977

19. Holmila M, Raitasalo K: Gender differences in drinking: why do they still exist? Addiction 100:1763-1769, 2005

20. Iseki C, Takahashi Y, Wada M, Kawanami T, Adachi M, Kato T: Incidence of idiopathic normal pressure hydrocephalus (iNPH): a 10-year follow-up study of a rural community in Japan. J Neurol Sci 339:108-112, 2014

21. Jacobs L: Diabetes mellitus in normal pressure hydrocephalus. J Neurol Neurosurg Psychiatry 40:331-335, 1977

22. Kazui H, Miyajima M, Mori E, Ishikawa M: Lumboperitoneal shunt surgery for idiopathic normal pressure hydrocephalus (SINPHONI-2): an open-label randomised trial. Lancet Neurol 14:585-594, 2015

23. Koto A, Rosenberg G, Zingesser LH, Horoupian D, Katzman $\mathrm{R}$ : Syndrome of normal pressure hydrocephalus: possible relation to hypertensive and arteriosclerotic vasculopathy. J Neurol Neurosurg Psychiatry 40:73-79, 1977

24. Krauss JK, Regel JP, Vach W, Droste DW, Borremans JJ, Mergner T: Vascular risk factors and arteriosclerotic disease in idiopathic normal-pressure hydrocephalus of the elderly. Stroke 27:24-29, 1996

25. Kristensen B, Malm J, Fagerland M, Hietala SO, Johansson B, Ekstedt J, et al: Regional cerebral blood flow, white matter abnormalities, and cerebrospinal fluid hydrodynamics in patients with idiopathic adult hydrocephalus syndrome. $\mathbf{J}$ Neurol Neurosurg Psychiatry 60:282-288, 1996

26. Leinonen V, Koivisto AM, Savolainen S, Rummukainen $\mathrm{J}$, Sutela A, Vanninen R, et al: Post-mortem findings in 10 patients with presumed normal-pressure hydrocephalus and review of the literature. Neuropathol Appl Neurobiol 38:72-86, 2012

27. Marmarou A, Young HF, Aygok GA: Estimated incidence of normal pressure hydrocephalus and shunt outcome in patients residing in assisted-living and extended-care facilities. Neurosurg Focus 22(4):E1, 2007

28. Montagne A, Barnes SR, Sweeney MD, Halliday MR, Sagare AP, Zhao Z, et al: Blood-brain barrier breakdown in the aging human hippocampus. Neuron 85:296-302, 2015

29. Mori K, Maeda M, Asegawa S, Iwata J: Quantitative local 
cerebral blood flow change after cerebrospinal fluid removal in patients with normal pressure hydrocephalus measured by a double injection method with $\mathrm{N}$-isopropyl-p-[123I] iodoamphetamine. Acta Neurochir (Wien) 144:255-263, 2002

30. Nixon PF, Jordan L, Zimitat C, Rose SE, Zelaya F: Choroid plexus dysfunction: the initial event in the pathogenesis of Wernicke's encephalopathy and ethanol intoxication. Alcohol Clin Exp Res 32:1513-1523, 2008

31. Qi Q, Liu X, Zhang G, He W, Ma R, Cong B, et al: Morphological changes of cerebral vessels and expression patterns of MMP-2 and MMP-9 on cerebrovascular wall of alcoholic rats. Int J Clin Exp Pathol 7:1880-1888, 2014

32. Ridley NJ, Draper B, Withall A: Alcohol-related dementia: an update of the evidence. Alzheimers Res Ther 5:3, 2013

33. Román GC: White matter lesions and normal-pressure hydrocephalus: Binswanger disease or Hakim syndrome? AJNR Am J Neuroradiol 12:40-41, 1991

34. Shukla D, Singh BM, Strobos RJ: Hypertensive cerebrovascular disease and normal pressure hydrocephalus. Neurology 30:998-1000, 1980

35. Strang NM, Claus ED, Ramchandani VA, Graff-Guerrero A, Boileau I, Hendershot CS: Dose-dependent effects of intravenous alcohol administration on cerebral blood flow in young adults. Psychopharmacology (Berl) 232:733-744, 2015

36. Tanaka N, Yamaguchi S, Ishikawa H, Ishii H, Meguro K: Prevalence of possible idiopathic normal-pressure hydrocephalus in Japan: the Osaki-Tajiri project. Neuroepidemiology 32:171-175, 2009

37. Tisell M, Tullberg M, Hellström P, Edsbagge M, Högfeldt M, Wikkelsö C: Shunt surgery in patients with hydrocephalus and white matter changes. J Neurosurg 114:1432-1438, 2011

38. Tolentino NJ, Wierenga CE, Hall S, Tapert SF, Paulus MP, Liu TT, et al: Alcohol effects on cerebral blood flow in subjects with low and high responses to alcohol. Alcohol Clin Exp Res 35:1034-1040, 2011
39. US Department of Agriculture, US Department of Health and Human Services: Dietary Guidelines for Americans 2010. Washington, DC: US Government Printing Office, 2010

40. Virhammar J, Laurell K, Cesarini KG, Larsson EM: Preoperative prognostic value of MRI findings in 108 patients with idiopathic normal pressure hydrocephalus. AJNR Am J Neuroradiol 35:2311-2318, 2014

\section{Disclosures}

The authors report no conflict of interest concerning the materials or methods used in this study or the findings specified in this paper.

\section{Author Contributions}

Conception and design: Johnson. Acquisition of data: Johnson, Hickman, Shuman, Johnson, F Yang, RR Rice, IM Rice, Chung, Wiemann, Tinl, Iracheta, Chen, Flynn, Mondello, Thompson, Carroll, HW Yang, Pilgrim, Chiocca, Dunn, Golby. Analysis and interpretation of data: Johnson, Hickman, Shuman, Tinl, Iracheta, Chen, Flynn. Drafting the article: Johnson, Hickman, Shuman. Critically revising the article: Johnson, Hickman, Shuman. Reviewed submitted version of manuscript: all authors. Approved the final version of the manuscript on behalf of all authors: Johnson. Statistical analysis: Johnson, Hickman, Shuman. Administrative/technical/material support: Johnson. Study supervision: Johnson.

\section{Correspondence}

Mark D. Johnson, Department of Neurosurgery, Harvard Medical School, Brigham and Women's Hospital, 75 Francis St., Boston, MA 02115. email: mjohnson27@partners.org. 